\title{
How to Build an Inexpensive 5-DOF Haptic Device Using Two Novint Falcons
}

\author{
Aman V. Shah, Scott Teuscher, Eric W. McClain, and Jake J. Abbott \\ Department of Mechanical Engineering, University of Utah \\ Salt Lake City, UT, 84112, USA \\ \{aman.shah, scott.teuscher, eric.mcclain, jake.abbott\}@utah.edu
}

\begin{abstract}
We demonstrate how two Novint Falcons, inexpensive commercially available haptic devices, can be modified to a create a reconfigurable five-degreeof-freedom (5-DOF) haptic device for less than $\$ 500$ (including the two Falcons). The device is intended as an educational tool to allow a broader range of students to experience force and torque feedback, rather than the 3-DOF force feedback typical of inexpensive devices. We also explain how to implement a 5-DOF force/torque control system with gravity compensation.
\end{abstract}

Keywords: Falcon, Torque Feedback, Gravity Compensation, Education.

\section{Introduction}

A number of commercially available haptic devices are available on the market. Inexpensive devices are limited to three degree-of-freedom (3-DOF) of force feedback, limiting users to point-like interactions, which places limits on the realism of haptic virtual environments. Haptic devices with 5- or 6-DOF of actuation exist-enabling both force and torque feedback - but cost many tens of thousands of dollars. One solution to this problem is to construct a 5-DOF haptic device by connecting two 3-DOF devices [1]. In this paper we demonstrate how two Novint Falcons-commercially available devices that costs less than $\$ 200$ each [2]—can be nondestructively modified to a create a reconfigurable 5-DOF device for less than an additional $\$ 100$. We explain how to implement gravity compensation on Falcons, and we show how to modify the two Falcon control systems to display a single 5-DOF force/torque. The complete instructions, mechanical drawings, and parts list to build the device shown in Fig. 1 can be found on the Utah Telerobotics Lab web page [3]. In addition, we have posted sample code implementing a 5-DOF controller with gravity compensation and a simple virtual environment using the open-source CHAI 3D software [4].

\section{Hardware}

Our modified 5-DOF device is shown in Fig. 1. The stylus is a simple rod with ball joints attached on each end. The concept behind the our device is to allow the same hardware to be reconfigured such that the stylus of the device can be either vertical or horizontal when the Falcons are in their respective zero positions, which roughly 


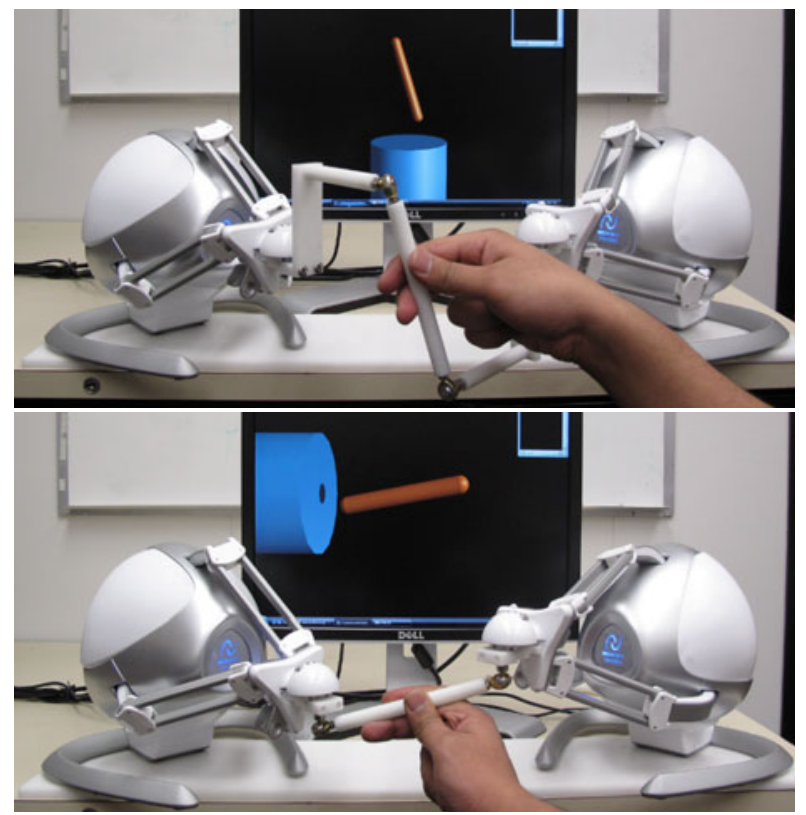

Fig. 1. Two Novint Falcon haptic devices modified for 5-DOF. The same hardware can be used in both a vertical (top) and horizontal (bottom) stylus configuration. The right-handed vertical configuration is shown; we find that a conflict between the user's hand and the device occurs between the hand and the upper extension piece. The device can be reversed for left-handed use.

correspond to the centers of their respective workspaces. Being able to reconfigure the stylus is desirable because of the limited workspace of the Falcons, which leads to limited angular movement of the stylus. The Falcons each apply a 3-DOF force to their respective ball joint, resulting in a total 3-DOF force, 2-DOF torque applied to the stylus. The stylus can rotate freely about its axis. The Falcons are mounted on a common baseplate, which is not strictly necessary, but is desirable because it keeps the Falcons properly aligned and provides some additional ballast. The Falcons are each rotated inward by $45^{\circ}$, such that they are perpendicular to one another. This rotation has the effect of (1) letting the stylus hang over the edge of the table to take advantage of the total workspace, (2) allowing the stylus to be a comfortable length while still allowing relatively large rotations of the stylus, (3) enabling simple outward extension pieces for the vertical-stylus configuration, and (4) keeping the overall width of combined 5-DOF device small. The length and diameter of the stylus, as well as the separation distance of the Falcons, were iteratively chosen based on comfort and range of motion. Collisions of the stylus with other components occur slightly beyond the workspace limits of the Falcons. Components were designed to be fabricated from common stock, which keeps the cost of the hardware low.

A Falcon will not function if the grip is removed, so any simple modification of a Falcon requires that the grip stay in place. Fortunately, the design of the grip facilitates simple modifications. After peeling away a thin backing, the lower hemisphere of the 


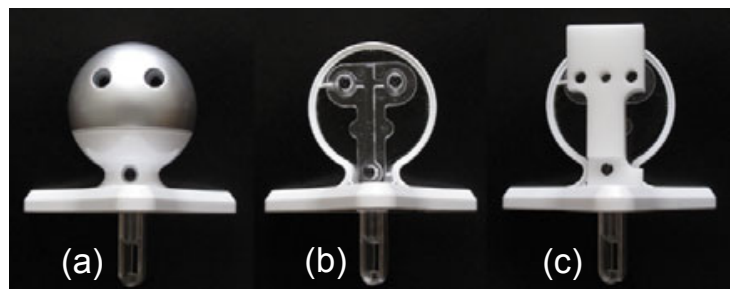

Fig. 2. Modification of the Falcon grip. (a) The original grip, shown from below. (b) Removing the lower hemisphere reveals a mounting plate. (c) A modified component can utilize the existing mounting plate.

grip can be removed, revealing a mounting plate that holds the circuitry for the grip's buttons, as shown in Fig.2(b). The screw holes in this mounting plate can be utilized for mounting additional hardware. The component added in Fig.2(c) is designed to allow the stylus to be attached in both the vertical and horizontal configurations shown in Fig. 1,

\section{Gravity Compensation}

After adding more mass to the already-heavy Falcon grip, it is desirable to implement gravity compensation, such that the Falcons will remain in any configuration in which they are placed, rather than falling under their own weight. We find that the fidelity of even a standard Falcon is noticeably improved when gravity compensation is implemented.

The kinematics of the Falcon are similar to the popular Delta mechanism, but are actually that of a modified mechanism designed at the University of Maryland [5]. A labeled photo of a Falcon along with a matching schematic of the kinematics is shown in Fig. 3. As described in [5], two Jacobian matrices relate velocities of the joints and the grip:

$$
J_{I} \dot{\theta}=J_{F} \dot{p}
$$

$$
\dot{\theta}=\left[\begin{array}{c}
\dot{\theta}_{11} \\
\dot{\theta}_{12} \\
\dot{\theta}_{13}
\end{array}\right], \dot{p}=\left[\begin{array}{l}
V_{p, x} \\
V_{p, y} \\
V_{p, z}
\end{array}\right], J_{F}=\left[\begin{array}{lll}
J_{F_{11}} & J_{F_{12}} & J_{F_{13}} \\
J_{F_{21}} & J_{F_{22}} & J_{F_{23}} \\
J_{F_{31}} & J_{F_{32}} & J_{F_{33}}
\end{array}\right], J_{I}=\left[\begin{array}{ccc}
J_{I_{1}} & 0 & 0 \\
0 & J_{I_{2}} & 0 \\
0 & 0 & J_{I_{3}}
\end{array}\right]
$$

$\theta_{j i}$ is angular displacement of joint $j$ on chain $i$ (joint 1 is actuated), $V_{p, x}$ is the velocity of the point $p$ in the center of the moving platform in direction $x$ of the Falcon's coordinate system ( $y$ is right, $z$ is up, and $x$ is toward the user), and

$$
\begin{gathered}
J_{F_{i 1}}=\cos \left(\theta_{2 i}\right) \sin \left(\theta_{3 i}\right) \cos \left(\phi_{i}\right)-\cos \left(\theta_{3 i}\right) \sin \left(\phi_{i}\right) \\
J_{F_{i 2}}=\cos \left(\theta_{3 i}\right) \cos \left(\phi_{i}\right)+\cos \left(\theta_{2 i}\right) \sin \left(\theta_{3 i}\right) \sin \left(\phi_{i}\right) \\
J_{F_{i 3}}=\sin \left(\theta_{2 i}\right) \sin \left(\theta_{3 i}\right) \\
J_{I_{i}}=a \sin \left(\theta_{2 i}-\theta_{1 i}\right) \sin \left(\theta_{3 i}\right)
\end{gathered}
$$



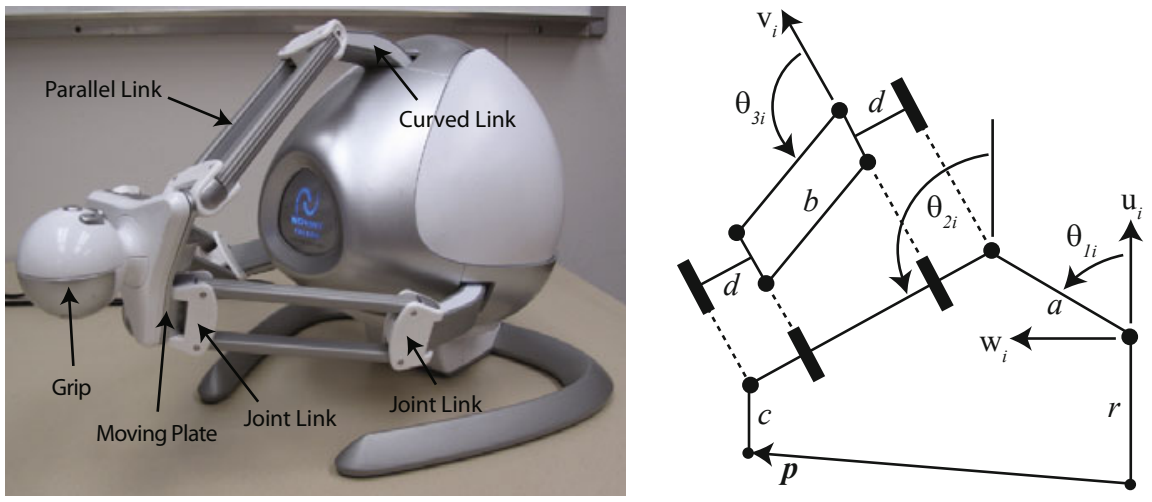

Fig. 3. Falcon kinematics based on [5]. Links $a$ and $b$ represent the curved and parallel links, respectively. The location of the center of the moving plate with respect to the origin is given by $p$. The distance from the origin to each actuated joint axis is $r$. The distance from the center of the moving plate to each of its joint axes is $c$. Links labeled $d$ represent the joint links, which are not present in a Delta mechanism.

The manipulator Jacobian for this closed-chain robot is computed as

$$
J=J_{I}^{-1} J_{F}
$$

The above calculation assumes knowledge of the joint angles, but this might not be available directly. For example, the CHAI 3D package only provides the position of a Falcon end-effector. Here we present a simplified version of the inverse kinematics of the general Maryland manipulator [5] as applied to the Falcon. The Falcon returns its coordinates as $\left[\begin{array}{lll}f_{x} & f_{y} & f_{z}\end{array}\right]^{T}$, and these coordinates must be converted to the base frame as $p_{x}=f_{y}, p_{y}=f_{z}$ and $p_{z}=f_{x}+p_{z, 0} \cdot p_{z, 0}$ and $p_{v, 0}$ are offset distances from the base plate to the center of the moving plate of the Falcon end-effector when the Falcon returns $f_{x}=f_{y}=f_{z}=0$. We convert the position of point $p$ to the local $u v w$ coordinate frame of each leg $i$, centered at its actuated joint (see Fig. 3) as:

$$
\left[\begin{array}{l}
p_{u i} \\
p_{v i} \\
p_{w i}
\end{array}\right]=\left[\begin{array}{ccc}
\cos \left(\phi_{i}\right) & \sin \left(\phi_{i}\right) & 0 \\
-\sin \left(\phi_{i}\right) & \cos \left(\phi_{i}\right) & 0 \\
0 & 0 & 1
\end{array}\right]\left[\begin{array}{l}
p_{x} \\
p_{y} \\
p_{z}
\end{array}\right]+\left[\begin{array}{c}
-r \\
p_{v, 0} \\
0
\end{array}\right]
$$

The expressions for $p_{u i}, p_{v i}$, and $p_{w i}$ are given by:

$$
\begin{gathered}
p_{u i}=a \cos \left(\theta_{1 i}\right)-c+\left(2 d+b \sin \left(\theta_{3 i}\right)\right) \cos \left(\theta_{2 i}\right) \\
p_{v i}=b \cos \left(\theta_{3 i}\right)+s \\
p_{w i}=a \sin \left(\theta_{1 i}\right)+\left(2 d+b \sin \left(\theta_{3 i}\right)\right) \sin \left(\theta_{2 i}\right)
\end{gathered}
$$

$s$ is the offset distance of each arm measured along the actuated-joint axis, which is not present in the Maryland manipulator. Solving (10) gives two values for $\theta_{3 i}$, but the 
Falcon uses only the positive value $\left(\theta_{3 i}\right.$ near $\left.90^{\circ}\right)$ :

$$
\theta_{3 i}=\cos ^{-1}\left(\frac{p_{v i}-s}{b}\right)
$$

Squaring and adding (9) and (11) gives

$$
\begin{gathered}
\left(p_{u i}+c\right)^{2}+p_{w i}^{2}+a^{2}-2 a\left(p_{u i}+c\right) \cos \left(\theta_{l i}\right)-2 a p_{w i} \sin \left(\theta_{l i}\right) \\
=4 d^{2}+4 d b \sin \left(\theta_{3 i}\right)+b^{2} \sin \left(\theta_{3 i}\right)^{2}
\end{gathered}
$$

Define a half-angle tangent as:

$$
t_{1 i}=\tan \left(\frac{\theta_{1 i}}{2}\right) \Rightarrow \sin \left(\theta_{1 i}\right)=\frac{2 t_{1 i}}{1+t_{1 i}^{2}}, \cos \left(\theta_{1 i}\right)=\frac{1-t_{1 i}^{2}}{1+t_{1 i}^{2}}
$$

Substitution into (13) gives

$$
\begin{gathered}
l_{2 i} t_{1 i}^{2}+l_{1 i} t_{1 i}+l_{0 i}=0 \\
l_{0 i}=p_{w i}^{2}+p_{u i}^{2}+2 c p_{u i}-4 d^{2}-b^{2} \sin ^{2}\left(\theta_{3 i}\right)-4 b d \sin \left(\theta_{3 i}\right)-2 a p_{u i}+(a-c)^{2} \\
l_{1 i}=-4 a p_{w i} \\
l_{2 i}=p_{w i}^{2}+p_{u i}^{2}+2 c p_{u i}-4 d^{2}-b^{2} \sin ^{2}\left(\theta_{3 i}\right)-4 b d \sin \left(\theta_{3 i}\right)+2 a p_{u i}+(a+c)^{2}
\end{gathered}
$$

Only one solution of (15) is applicable to the Falcon (due to the limited range of $\theta_{1 i}$ ), which is used with (14) to solve for $\theta_{1 i}$ :

$$
t_{1 i}=\frac{-l_{1 i}-\sqrt{l_{1 i}^{2}-4 l_{2 i} l_{0 i}}}{2 l_{2 i}} \Rightarrow \theta_{1 i}=2 \tan ^{-1}\left(t_{1 i}\right)
$$

Finally, combining (9) and (11) gives:

$$
\theta_{2 i}=\tan ^{-1}\left(\frac{p_{w i}-a \sin \left(\theta_{1 i}\right)}{p_{u i}-a \cos \left(\theta_{1 i}\right)+c}\right)
$$

Once we are equipped with a manipulator Jacobian and inverse kinematics, we are ready to implement gravity compensation. The joint torques $\tau_{g}=\left[\begin{array}{lll}\tau_{1} & \tau_{2} & \tau_{3}\end{array}\right]^{T}$ for gravity compensation are

$$
\begin{aligned}
\tau_{g}= & g m_{a} q\left[\begin{array}{l}
\sin \left(100^{\circ}-\theta_{11}\right) \sin \left(\phi_{1}\right) \\
\sin \left(100^{\circ}-\theta_{12}\right) \sin \left(\phi_{2}\right) \\
\sin \left(100^{\circ}-\theta_{13}\right) \sin \left(\phi_{3}\right)
\end{array}\right]-g\left(m_{b}+m_{d}\right) a\left[\begin{array}{l}
\sin \left(\theta_{11}\right) \sin \left(\phi_{1}\right) \\
\sin \left(\theta_{12}\right) \sin \left(\phi_{2}\right) \\
\sin \left(\theta_{13}\right) \sin \left(\phi_{3}\right)
\end{array}\right] \\
& +g\left(3\left(m_{b}+m_{d}\right)+m_{c}+m_{e}\right) J^{-T}\left[\begin{array}{lll}
0 & 1 & 0
\end{array}\right]^{T}
\end{aligned}
$$


where the mass labeling corresponds to Fig. 3, $g=9.81$ is the acceleration of gravity, and $m_{e}$ is the mass of the end-effector. In the case of a standard Falcon, $m_{e}$ is the mass of the detachable grip. In the case of of our 5-DOF device, $m_{e}$ includes the mass of the modified grip of Fig.2 (2), as well as $50 \%$ of the mass of the additional added hardware (the two Falcons equally share the load of the new hardware). When using a Falcon, the user does not command motor torques, but rather, commands forces to the grip. The equivalent gravity-compensation force is computed as $f_{g}=J^{T} \tau_{g}$. In Table 1 we report important mass and length parameters, which we measured from a disassembled Falcon. Additional Falcon characterization can be found in [6].

Gravity compensation for the Maryland manipulator is given in [5], so we followed that derivation here, making two key modification for use with a Falcon. First, the Falcon sits on its side, making gravity loads unequal on each of the three motors, as opposed to the vertical Maryland manipulation. This leads to the terms that include the $\phi$ 's in 21. The Falcon's three actuated joints lie in a vertical back plane. We experimentally measured these axes to be described by $\phi_{1}=105^{\circ}, \phi_{2}=-15^{\circ}$, and $\phi_{3}=$ $-135^{\circ}$, measured about $z$ from $y$ in the base frame such that $\phi=0$ corresponds to aligned $u v w$ and $x y z$ frames. Second, the mass distri-

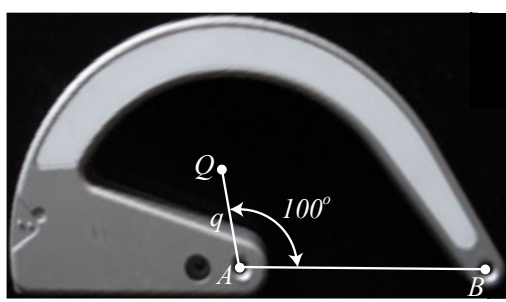

Fig. 4. Curved first link of the Falcon. The center of mass was experimentally determined to be at the point $Q$, with $q=22 \mathrm{~mm}$ bution in the first link of the Falcon is significantly different than that of the Maryland robot [5], where the center of mass of the first link is at the midpoint between the two joints $A$ and $B$. As shown in Fig. 4 , we experimentally found the center of mass of the Falcon's first link to be far from that midpoint, leading to the $q$ and $100^{\circ}$ terms in (21).

Table 1. Measured mass and length parameters

\begin{tabular}{|c|c|c|}
\hline Part Name & Mass (kg) & Length (m) \\
\hline Curved Link & $m_{a}=0.089$ & $a=0.060$ \\
Parallel Link & $m_{b}=0.008$ & $b=0.103$ \\
Moving Plate & $m_{c}=0.033$ & $c=0.016$ \\
Joint Link & $m_{d}=0.010$ & $d=0.011$ \\
Back Plate Radius & - & $r=0.037$ \\
Back Plate Actuator Offset & - & $s=0.025$ \\
Zero-Configuration z-Offset & - & $p_{z, 0}=0.134$ \\
Zero-Configuration -Offset & - & $p_{v, 0}=0.022$ \\
Standard Falcon Grip (Fig.[2]a)) & $m_{e}=0.052$ & - \\
\hline Stylus, From Centers of Ball Joints & - & $l_{S}=0.166$ \\
$50 \%$ Vertical Stylus Assembly (Fig.11a)) & $m_{e}=0.128$ & - \\
$50 \%$ Horizontal Stylus Assembly (Fig.[1]b)) & $m_{e}=0.083$ & - \\
\hline
\end{tabular}




\section{The 5-DOF Controller}

The final step is to coordinate the individual 3-DOF forces of the Falcons such that the user experiences a single 5-DOF device. We define a world coordinate system such that when both Falcons are at their respective zero positions, the stylus is at its zero position in the world coordinate frame. The world frame is such that stylus movements in the $y$ direction are to the right, $z$ is up, and $x$ is toward the user. The positions of interest kinematically are those of the two ball joints on the ends of the stylus, so when we refer to the position of, say, the left Falcon, we are referring to the position of the center of its ball joint. The homogeneous transformation that relates the position $p_{i}$ of Falcon $i$ in its own frame to the world frame is

$$
\left[\begin{array}{c}
{ }^{w} p_{i} \\
1
\end{array}\right]=\left[\begin{array}{cccc}
\cos (\alpha) & -\sin (\alpha) & 0 & 0 \\
\sin (\alpha) & \cos (\alpha) & 0 & \beta \\
0 & 0 & 1 & \gamma \\
0 & 0 & 0 & 1
\end{array}\right]\left[\begin{array}{c}
{ }^{i} p_{i} \\
1
\end{array}\right]
$$

There are three different arrangements of stylus, two of which are shown in Fig. 1] vertical right-handed, vertical left-handed, and horizontal. The arrangement-specific parameters for (22) are given in Table 2, where $l_{S}$ is the length of the stylus measured from the centers of the ball joints.

Our goal is to display an effective force and torque at a single point $p_{S}$ somewhere along the length of the stylus. We first define a vector $\Delta$ from the left Falcon position (ball joint) to the right Falcon position:

$$
\Delta=p_{R}-p_{L}
$$

Then we define $p_{S}$ relative to $p_{L}$ :

$$
p_{S}=p_{L}+\delta \Delta
$$

Choosing $\delta=0$ sets $p_{S}=p_{L}, \delta=1$ sets $p_{S}=p_{R}$, and $\delta=0.5$ sets $p_{S}$ at the center of the stylus. By choosing $\delta<0$ or $\delta>1$, we can place the effective stylus point outside of the physical stylus.

To display a pure force $f_{S}$ at the stylus point $p_{S}$, we decompose the force into individual right and left Falcon forces as

$$
f_{R f}=\delta f_{S}, \quad f_{L f}=(1-\delta) f_{S}
$$

Table 2. Coordinate-frame parameters for different arrangement of the stylus

\begin{tabular}{|c|c|c|}
\hline Parameters & Left Falcon & Right Falcon \\
\hline \hline$\alpha$ & $45^{\circ}$ & $-45^{\circ}$ \\
$\beta$ - Horizontal Stylus & $-l_{S} / 2$ & $l_{S} / 2$ \\
$\gamma$ - Horizontal Stylus & 0 & 0 \\
$\beta$ - Vertical Right-Handed Stylus & 0 & 0 \\
$\gamma$ - Vertical Right-Handed Stylus & $l_{S} / 2$ & $-l_{S} / 2$ \\
$\beta$ - Vertical Left-Handed Stylus & 0 & 0 \\
$\gamma$ - Vertical Left-Handed Stylus & $-l_{S} / 2$ & $l_{S} / 2$ \\
\hline
\end{tabular}


To display a pure torque $\tau_{S}$ at the stylus point $p_{S}$, we apply individual Falcon forces as

$$
f_{R \tau}=\frac{1}{l_{S}^{2}}\left(\tau_{S} \times \Delta\right), \quad f_{L \tau}=-f_{R \tau}
$$

To display an arbitrary force/torque, the pure force and pure torque simply sum before being commanded to the Falcons:

$$
f_{R}=f_{R f}+f_{R \tau}, \quad f_{L}=f_{L f}+f_{L \tau}
$$

The above equations assume all forces and torques are described with respect to the world frame. The left and right Falcon forces must be transformed into their respective frames, which is accomplished using the inverse of the transformation matrix in (22). The final step is to sum the forces from this 5-DOF controller with the gravitycompensation forces of Section 3 such that a single force vector can be commanded to each of the Falcons.

\section{Conclusion}

Many students who are exposed to haptics only get to experience inexpensive 3-DOF devices. Using the steps outlined in this paper, a student can build a 5-DOF device by nondestructively modifying two Novint Falcons for less than an additional \$100. This 5-DOF device will allow more students to get to experience realistic force/torque interactions with virtual environments. In this paper, we designed a simple 5-DOF modification mechanism, we summarized the manipulator Jacobian and inverse kinematics for a Novint Falcon, we derived a gravity compensation algorithm that applies to both a standard Falcon as well as the 5-DOF system presented here, and we provided a number of useful length and mass parameters obtained empirically by destructively disassembling a Falcon. Additional details can be found on the Utah Telerobotics Lab web page [3].

\section{References}

1. Martín, J., Savall, J.: Mechanisms for haptic torque feedback. In: Proc. Joint Eurohaptics Conf. and Symp. Haptic Interfaces for Virtual Environment and Teleoperator Systems, pp. 611-614 (2005)

2. Novint Falcon, http://home.novint.com/products/novint_falcon.php

3. The Univerisity of Utah Telerobotics Lab, http://www.telerobotics.utah.edu

4. CHAI 3D: The open source haptics project, http://www.chai3d.org/

5. Stamper, R.E.: A Three Degree of Freedom Parallel Manipulator with Only Translational Degrees of Freedom. PhD thesis, Department of Mechanical Engineering, The University of Maryland (1997)

6. Martin, S., Hillier, N.: Characterisation of the Novint Falcon haptic device for application as a robot manipulator. In: Proc. Australasian Conf. Robotics and Automation (2009) 